knowledge levels of sunscreen creams, sun protection factor (SPF), ultraviolet A (UVA) and ultraviolet B (UVB), it was determined that $11.2 \%$ of the participants knew the definition of SPF 30. In the question about UVA and UVB, 27.9\% answered correctly.

Discussion The use of sun protective cream among the sun protection methods among the academic staff was found to be the first place. However, it has been determined that this method of protection is not conscious and orderly. Academic staff's knowledge of sunscreen creams, SPF, UVB and UVA was found to be inadequate. Therefore, we believe that future sun protection programmes could target in order to improve academic staff's knowledge and attitudes towards sun protection, effects of the sun on skin and skin cancer.

\section{OCCUPATIONAL RADIATION EXPOSURE TO PREGNANT HEALTHCARE WORKERS}

Hassan A AlGhamdi, Moazzam Zaidi*, Ezzat Mansour, Hussam Saeed. Johns Hopkins Aramco Healthcare (JHAH). ${ }^{2} \mathrm{HHAH}$

\subsection{6/oemed-2018-ICOHabstracts. 1223}

Introduction Radiation exposure to healthcare workers is a known occupational hazard. With a significant number of female healthcare employees of childbearing age in the workforce, it is essential to ensure that occupational exposure is at the lowest possible limits. The fetus is sensitive to high doses of ionising radiation. The probability that a particular effect will occur depends on several factors, including dose, stage of gestation, and dose rate. A number of strategies and administrative controls are used to protect healthcare workers.

Method JHAH has an active radiation protection program for pregnant radiation workers. The program includes training, exposure monitoring and record review, modifications to working conditions, protective devices and administrative procedures to control exposure. The JHAH Radiation Protection Team provides individual case-based risk assessments and, if required, work condition modifications and necessary administrative procedures are discussed. In addition, occupational medicine provides advice and counselling as required. Two case studies will be discussed showing how the JHAH Radiation Protection program works.

Results Assessments have shown effective use of time, distance and shielding resulted in exposures well below advised guidelines. Additional radiation monitors worn at the abdomen level of pregnant healthcare workers resulted in enhanced fetus monitoring. In cases assessed, readings have been found below the level of public exposure.

Discussion/conclusion The JHAH Radiation Protection program demonstrates that proactive measures and regular monitoring provides healthcare workers with reassurance that exposure is well below exposure limits. Risk assessment for pregnant healthcare workers at work provides further reassurance that employees and their fetuses are safe. This psychological assurance has made a positive contribution to increased morale and satisfaction of the healthcare workers.

It is important to establish radiation guidelines and training programs for pregnant radiation healthcare workers.
1283 GENE EXPRESSION ANALYSIS OF BLOOD CELLS IN RADIATION HEALTH CARE WORKERS OCCUPATIONALLY EXPOSED TO IONISING RADIATION

${ }^{1} \mathrm{R}$ Brugaletta ${ }^{*},{ }^{1,2} \mathrm{~A}$ Santoro, ${ }^{3} \mathrm{~A}$ Alisi, ${ }^{3} \mathrm{~N}$ Panera, ${ }^{4} \mathrm{~A}$ Pastore, ${ }^{4} \mathrm{G}$ Di Giovamberardino, 1,2MR Vinci, 1,2V Camisa, 'L Lavorato, ${ }^{1,2} 2 \mathrm{~S}$ Zaffina. ${ }^{1}$ Health Technology Assessment and Safety Research Unit, Bambino Gesù Children's Hospital IRCCS, Rome, Italy; ${ }^{2}$ Occupational Health Service, Bambino Gesù Children's Hospital IRCCS, Rome, Italy; ${ }^{3}$ Liver Research Unit, Bambino Gesù Children's Hospital IRCCS, Rome, Italy; ${ }^{4}$ Research Genetics and Rare Diseases Unit, Bambino Gesù Children's Hospital IRCCS, Rome, Italy

\subsection{6/oemed-2018-ICOHabstracts.1224}

Introduction Ionising radiation (IR) is a ubiquitous environmental agent whose effects on organism are well established. A direct interaction of IR with human cells causes a variety of biological effects including neoplastic transformation. In this context, physical and biological dosimetry could be a key tool for individual monitoring of the absorbed IR. However, since the current dosimetry approaches are not tailored to distinguish the early response and long-term pro-oncogenic effects of low-dose (LD)IR the discovery of inherent biomarkers represents a priority area. We hypothesised that changes of gene expression profiles in blood cells may occur during occupational exposure to LDIR and that the identification of these changes could be potentially useful in the early recognition of pro-oncogenic phenotypes.

Methods We included 9 workers exposed to IR and 3 nonexposed workers, all equipped with dosimeter. Exclusion criteria were smoking, history of cancer diagnosis before occupational exposure, high blood pressure medication and intake of paracetamol. Blood cells were collected from each worker and used to evaluate transcriptional expression of a panel of 624 cancerrelated genes by using the Real-Time PCR OpenArray technology.

Results The analysis in radiation workers exposed showed the over-expression of some genes involved in the progression of certain types of tumours and an evident alteration in the expression of the gene involved in a way of regulation, known to predispose to genomic instability and tumorigenesis induced by IR. Intriguingly, we also found that among radiation workers 3 subjects, with occupationally recognised thyroid cancer displayed an up-regulation of the rearranged during transfection (RET) gene, which has been found previously overexpressed in subjects with radiation-induced thyroid cancers.

Discussion Our preliminary data would indicate that exposure to LDIR is able to alter the expression of pro-tumour genes in blood cells, posing questions and opening new scenarios towards a 'personalised radioprotection' model.

\section{GENOTOXIC DAMAGE AND DNA REPAIR GENE POLYMORPHISMS IN WORKERS EXPOSED TO LOW DOSES OF IONISING RADIATION}

${ }^{1} \mathrm{P}$ Lovreglio*, ${ }^{1} \mathrm{R}$ Rotondi, ${ }^{1} \mathrm{P}$ Chiarappa, ${ }^{2} \mathrm{~N}$ Resta, ${ }^{2} \mathrm{R}$ Bagnulo, ${ }^{1} \mathrm{I}$ Drago, ${ }^{3} \mathrm{ME}$ Fracasso, ${ }^{3} \mathrm{D}$ Doria, ${ }^{1} \mathrm{~L}$ Soleo. ${ }^{1}$ Interdisciplinary Department of Medicine, Section of Occupational Medicine 'E.C. Vigliani', University of Bari, Italy; ${ }^{2}$ Department of Biomedical Sciences and Human Oncology, Division of Medical Genetics, University of Bari, Italy; ${ }^{3}$ Department of Medicine and Public Health, Section of Pharmacology, University of Verona, Italy

10.1136/oemed-2018-ICOHabstracts. 1225 